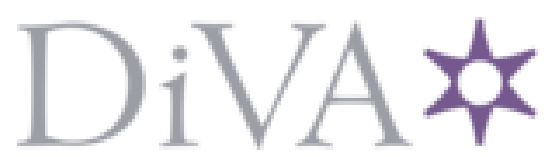

http://www.diva-portal.org

This is the published version of a paper published in Plant Biotechnology Journal.

Citation for the original published paper (version of record):

Hansson, C., Schön, K., Kalbina, I., Strid, Å., Andersson, S. et al. (2016)

Feeding transgenic plants that express a tolerogenic fusion protein effectively protects against arthritis.

Plant Biotechnology Journal, 14(4): 1106-1115

http://dx.doi.org/10.1111/pbi.12479

Access to the published version may require subscription.

N.B. When citing this work, cite the original published paper.

Permanent link to this version:

http://urn.kb.se/resolve?urn=urn:nbn:se:oru:diva-45625 


\title{
Feeding transgenic plants that express a tolerogenic fusion protein effectively protects against arthritis
}

\author{
Charlotta Hansson ${ }^{1}$, Karin Schön ${ }^{1}$, Irina Kalbina ${ }^{2}$, Åke Strid ${ }^{2}$, Sören Andersson ${ }^{2,3}$, Maria I. Bokarewa ${ }^{4}$ and
} Nils Y. Lycke ${ }^{1, *}$

${ }^{1}$ Department of Microbiology and Immunology, University of Gothenburg, Gothenburg, Sweden

${ }^{2}$ Örebro Life Science Center, School of Science and Technology, Örebro University, Örebro, Sweden

${ }^{3}$ Department of Laboratory Medicine, Örebro University hospital, Örebro, Sweden

${ }^{4}$ Department of Rheumatology and Inflammation Research, University of Gothenburg, Gothenburg, Sweden

Received 19 May 2015;

revised 27 July 2015;

accepted 21 August 2015

*Correspondence (Tel 0046(0)317866321;

Fax 0046(0)317862150; email

nils.lycke@microbio.gu.se)

Keywords: autoimmunity, transgenic plants, edible plants, CIA, IL-10, FoxP3.

\begin{abstract}
Summary
Although much explored, oral tolerance for treatment of autoimmune diseases still awaits the establishment of novel and effective vectors. We investigated whether the tolerogenic CTA1 (R7K)-COL-DD fusion protein can be expressed in edible plants, to induce oral tolerance and protect against arthritis. The fusion protein was recombinantly expressed in Arabidopsis thaliana plants, which were fed to $\mathrm{H}-2^{q}$-restricted DBA/1 mice to assess the preventive effect on collageninduced arthritis (CIA). The treatment resulted in fewer mice exhibiting disease and arthritis scores were significantly reduced. Immune suppression was evident in treated mice, and serum biomarkers for inflammation as well as anticollagen IgG responses were reduced. In spleen and draining lymph nodes, $C D 4^{+} \mathrm{T}$-cell responses were reduced. Concomitant with a reduced effector T-cell activity with lower IFN $\gamma$, IL-13 and IL-17A production, we observed an increase in IL-10 production to recall antigen stimulation in vitro, suggesting reduced Th1, Th2 and Th17 activity subsequent to up-regulated IL-10 and regulatory T-cell (Treg) functions. This study shows that edible plants expressing a tolerogen were effective at stimulating CD4 T-cell tolerance and in protecting against CIA disease. Our study conveys optimism as to the potential of using edible plants for oral treatment of rheumatoid arthritis.
\end{abstract}

\section{Introduction}

Rheumatoid arthritis (RA) disease, as well as other autoimmune conditions, is treated with anti-inflammatory drugs that ameliorate symptoms and reduce tissue destruction, but do not offer cure to the disease (Ichim et al., 2008; Smolen and Aletaha, 2015). The exceptions to current therapies involve experimental attempts to cure disease by remodelling specific immune responses and, hence, controlling the attack on tissue functions (Ichim et al., 2008). Mucosal tolerance and, in particular, oral tolerance are well-established therapies to treat autoimmune diseases in experimental models, but few clinical trials have successfully shown a significant effect on human disease (Barnett et al., 1996, 1998; Cazzola et al., 2000; Choy et al., 2001; Trentham et al., 1993). The reason for these failures are thought to depend on the requirement for high and multiple doses of antigen or the fact that no clinically acceptable immunomodulation has been found that impose T-cell tolerance on the immune system (Faria and Weiner, 2006).

We have previously demonstrated that the patented CTA1 (R7K)-COL-DD fusion protein is an effective tolerance-inducing vector, preventing and greatly reducing collagen-induced arthritis (CIA) disease following intranasal administration in mice (Hasselberg et al., 2009). The mutant CTA1(R7K) moiety is derived from the $A 1$ subunit of cholera toxin $(C T)$, but lacks enzymatic activity due to a single amino acid substitution. The CTA1(R7K) molecule was genetically fused to a dimer of the $D$ fragment of Staphylococcus aureus protein A, to target antigen-presenting cells (APC) (Hasselberg et al., 2010). These elements flank the immunodominant collagen type II (CII) peptide, amino acids
259-274 (COL), strongly associated with RA, and which effectively stimulates $\mathrm{CD}^{+}{ }^{+} \mathrm{T}$ cells in $\mathrm{H}-2^{\mathrm{q}} \mathrm{DBA} / 1$ mice (Trentham et al., 1977). Thus, the complex carries both APC targeting properties (DD) and immunomodulation through the mutant CTA1(R7K) moiety as well as the dominant species conserved peptide involved in RA disease development.

Most studies today ascribe the tolerogenic effects of mucosal antigen administration to the stimulation of regulatory CD4 T cells (Tregs) (Weiner et al., 2011). The generation of antigenspecific CD4 Tregs is a powerful strategy to reinstate tolerance in the immune system. The CTA1(R7K)-COL-DD tolerogen given intranasally stimulated Tregs and the mechanism of action appeared to be IL-10 dependent (Hasselberg et al., 2009, 2010). A major challenge to developers of mucosal tolerogenic therapies today is to identify a strategy for effective and sufficient delivery of antigen. Because nasal vaccine delivery has been met with scepticism by regulatory authorities, the use of other mucosal delivery routes have been favoured also for toleranceinducing therapies (Jabbal-Gill, 2010). Whereas our fusion protein was ineffective when given orally in the CIA model and large quantities of soluble antigen are usually required for a tolerogenic effect, we explored alternative strategies to achieve an effective and highly compliant therapy for RA patients. Many investigators have turned to edible plants as a potential approach to achieve mucosal tolerance against autoimmune diseases, especially for type I diabetes and, to some extent, also for RA (Arakawa et al., 1998; Hashizume et al., 2008; Ma et al., 1997; Ruhlman et al., 2007). Plants are unlikely carriers of human pathogens and provide natural bioencapsulation of the antigen, which will prevent protein degradation in the gut and allow for effective 
uptake in the gut-associated lymphoid tissue (GALT) (Limaye et al., 2006). Although oral tolerance has proven difficult to achieve with plants expressing disease-relevant recombinant proteins or peptides only, the combination with mucosal targeting using expression systems exploiting the potential of cholera Bsubunit (CTB) bound peptides have shown more promise. Examples of this strategy are lettuce or potatoes that recombinantly expressed human proinsulin-CTB, which had some reducing effects on disease in the experimental models (Arakawa et al., 1998; Ruhlman et al., 2007). This argues in favour of new and more effective tolerance-inducing components to be included in edible plants to make this approach clinically feasible.

RA is an autoimmune disease affecting approximately $1 \%$ of the population worldwide with symptoms that are chronic and incapacitating due to cartilage destruction and bone erosion in peripheral joints. It poses a significant health problem and economic challenge not only to Western societies but RA is also a growing problem in developing countries (Brooks, 2006; Okada et al., 2010). In RA, disease severity correlates with increased proinflammatory cytokines such as interferon- $\gamma($ IFN- $\gamma)$, interleukin- 6 (IL-6) and IL-17, as well as auto-aggressive CD4 T-cell infiltrates in the synovium (Firestein, 2003; McInnes and Schett, 2007). The broadly immunosuppressive biological agents used today are expensive, ineffective in a significant proportion of patients and often associated with side effects, such as increased risk of infections and cancer development (Beyaert et al., 2013; Bongartz et al., 2006; Salliot et al., 2009). This makes more effective and targeted therapies against RA with fewer side effects highly warranted.

This study was undertaken to investigate whether the tolerogenic CTA1(R7K)-COL-DD protein could be expressed in edible plants and enable the effective use of a targeted immunomodulating tolerogen for oral treatment of RA. We transformed Arabidopsis thaliana plants to express the tolerogenic CTA1(R7K)COL-DD protein and fed mice that were immunized with $\mathrm{Cll}$ to develop CIA disease. The results obtained clearly support the notion that edible plants can be used for tolerance treatment of autoimmune diseases, in general, and they offer promise as to the possibility to develop a disease-targeted oral therapy for RA, in particular.

\section{Results}

\section{Arabidopsis thaliana plant expression of the CTA1(R7K)-COL-DD tolerogen}

We genetically modified edible plants to express the toleranceinducing gene fusion protein that we previously have demonstrated could protect against arthritis in the collagen-induced arthritis (CIA) model after intranasal administration (Hasselberg et al., 2009). Here, our aim was to develop an effective oral therapy for inducing tolerance and prevent CIA disease. Therefore, we incorporated the tolerogen encoding CTA1(R7K)-COL-DD genetic construct that carries the $\mathrm{H}-2^{9}$-restricted collagen type II (CII) peptide 259-274 (COL) into the pGreen vector, which was then used for nuclear transformation of $A$. thaliana plants of the Col-0 ecotype (Figure 1a). After initial screening for successful transformation with BASTA (Clough and Bent, 1995), transgenic lines were identified and used for further analysis. Stable integration of the transgene (TG) and expression of the recombinant protein was monitored for up to six generations, and $\alpha$-CTA1-DD antibodies were used to detect a protein of the correct size $(\sim 39 \mathrm{kDa})$ by Western blot (Figure $1 \mathrm{~b})$. A semiquantitative analysis of the recombinant fusion protein content in the plant showed an expression of approximately $30 \mathrm{ng}$ of CTA1(R7K)-COL-DD protein per mg of $A$. thaliana biomass, or $25 \mathrm{ng}$ per $\mu \mathrm{g}$ of total soluble protein (TSP), corresponding to $2.5 \%$ of TSP (Figure 1C). The fusion protein CTA1(R7K)-COL-DD was detected in all plant organs with highest concentrations in the inflorescence and in the leaves (Figure 1d). A subfractionation analysis showed that as expected, no fusion protein was detected in the chloroplast fraction. Instead, it was mainly found in the cytosolic fraction, but with some protein retained in the insoluble fraction that could include cell debris and inclusion bodies (Figure 1e).

\section{Therapeutic effects on $\mathrm{CIA}$ of feeding transgenic Arabidopsis thaliana plants}

The CIA model is considered the best experimental model for RA, and it is frequently used to evaluate the efficacy of clinically relevant anti-inflammatory treatments (Brand et al., 2003). Therefore, DBA/1 mice were used to initiate CIA disease and a protocol for weekly feedings of either TG or control Arapidopsis thaliana plants was established. Control plants were either untransformed wild type (WT) or transformed with empty vector ( $p$ Green) or peptide-free fusion protein (CTA1R7K-DD). In each cage, 10 mice were allowed to feed ad libitum on the plants, which were served 1 day of the week for 4 weeks (Figure 2a). Based on a semiquantitative analysis of protein expression (30 ng/ mg of biomass), we estimated that each mouse would have sufficient amount of whole plant material to ingest approximately $200 \mu \mathrm{g}$ of fusion protein and, thus, $10 \mu \mathrm{g}$ of COL-peptide on each feeding occasion. Mice were monitored for CIA and, whereas $10-20 \%$ of the treated mice did not develop disease at all, the majority of mice feed TG plants exhibited significantly reduced CIA severity $(P<0.05)$, as opposed to mice fed WT plants, vector ( $p$ Green) plants or controls plants CTA1(R7K)-DD, not expressing the COL-peptide (Figure 2b,c).

\section{Protective mechanisms against $\mathrm{CIA}$ disease by feeding transgenic plants}

Next, we investigated the immune mechanisms that were responsible for the protective effects of feeding TG plants on $\mathrm{CIA}$ disease development. To this end, we analysed humoral as well as cell-mediated immune responses and focused attention on evidence of CD4 T-cell tolerance, as our previous studies using the CTA1(R7K)-COL-DD tolerogen in soluble form indicated strong induction of peptide-specific regulatory CD4 T cells (Hasselberg et al., 2009, 2010). We found that TG plant-treated mice had lower levels of anti-Cll protein $\lg G 1$ and $\operatorname{lgG} 2 \mathrm{a} / \mathrm{b}$ antibodies in serum than control mice fed WT plants, suggesting that both Th1 and Th2 responses were impaired in TG plant fed mice (Figure 3a). Furthermore, serum levels of MMP-3, reflecting cartilage and bone destruction, and which is strongly upregulated in CIA disease (Seeuws et al., 2010), were significantly reduced in TG plant fed mice as compared to the levels observed in the control mice that developed more severe CIA. In fact, the level of serum MMP-3 was not significantly higher in TG plant fed mice than that found in normal naive mice (Figure 3b). In addition, we found a significant reduction of the levels of serum IL-6 in TG plant fed mice as compared to those detected in control mice with CIA disease (Figure 3c), which is reminiscent of our previous observations with the CTA1(R7K)-COL-DD fusion protein. Taken together, these results indicated that TG plant feeding resulted in the suppression of systemic inflammatory parameters, indicating a preventive effect on CIA disease devel- 
(a)

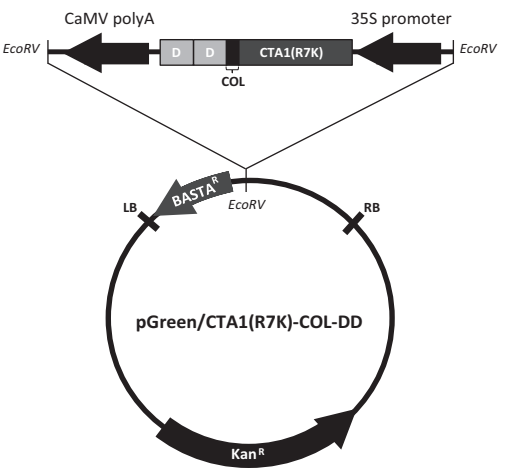

(b)

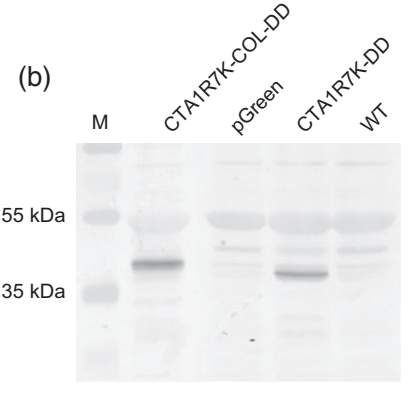

(d)

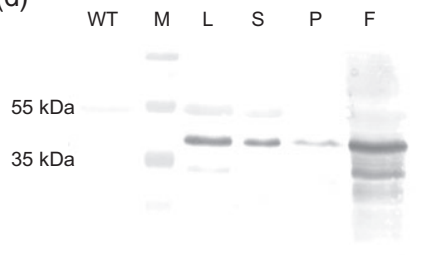

(c)
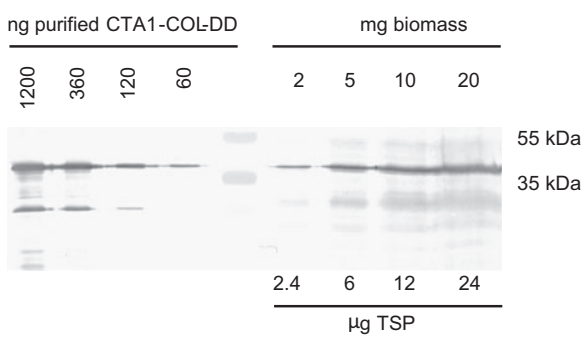

(e)

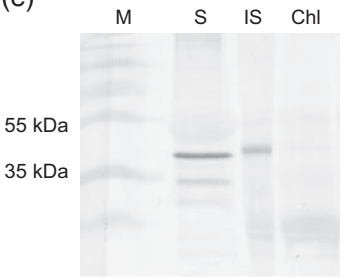

Figure 1 Arabidopsis thaliana plant expression of the CTA1(R7K)-COL-DD tolerogen. Schematic diagrams of the $\mathrm{pGreen}$ transformation vector and integration cassette/construct. Left border (LB) and right border (RB) flank the transfer DNA (t-DNA) integrated into the nuclear genome of A. thaliana (a). Western blot analysis of transgenic plants expressing CTA1(R7K)-COL-DD (1), empty vector pGreen (2), CTA1(R7K)-DD (3) or untransformed WT (4) using anti-CTA1-DD antibodies with positive bands at $\sim 39 \mathrm{kDa}$ (b). Expression levels of CTA1(R7K)-COL-DD in the TG plant were estimated by a semiquantitative analysis using recombinant CTA1(R7K)-COL-DD (C). Protein expression levels in different parts of TG plants were assessed in leaves (L), stems (S), siliques (P) and inflorescence (F). Protein was extracted with $50 \mathrm{~mm}$ Tris buffer ( $\mathrm{pH} 7.5$ ) containing $8 \mathrm{~m}$ urea. Extracts were $1 \mathrm{mg}$ biomass/ $\mu \mathrm{L}$ and $15 \mu \mathrm{L}$ of each sample was loaded onto the gel. (d). Furthermore, the following subcellular fractions were examined for their content of CTA1 (R7K)-COL-DD; soluble cytoplasmic (S), insoluble (IS) and chloroplast (Chl) fractions (e). opment. Because such a global suppressive effect on inflammation could be ascribed to the stimulation of regulatory CD4 T cells, we analysed the recall response to COL-peptide in CD4 T cells from spleen or draining popliteal lymph nodes in TG plant fed mice. We observed significantly reduced cell proliferation and supernatants demonstrated strikingly lower levels of IL-1 $\alpha$, IL-6, IFNy, IL-17 and GM-CSF as compared with CD4 T cells from control mice (Figure 4a). Of note, also IL-13 levels were reduced, supporting the notion that the suppressive effects we observed were not due to Th2-skewing of the COL-specific response. By contrast, IL-10 levels were enhanced in culture supernatants from CTA1(R7K)-COL-DD fed mice as compared to supernatants from control mice (Figure 4b). Hence, feeding tolerogenic TG plants stimulated regulatory CD4 T cells that produced IL-10, which strongly suppressed the expansion and/or function of COLspecific auto-aggressive $T$ cells and significantly impaired disease progression in the CIA model. This interpretation was further supported by the increase in circulating FoxP3 ${ }^{+}$Tregs in peripheral blood of TG plant fed mice, but which was not seen in mice fed WT plants (Figure 4c).

\section{Feeding transgenic plants protects against disease and tissue destruction}

Finally, we examined tissue sections of joints from CIA mice with or without feeding of the TG plants to assess the degree of tissue destruction. We found significantly less joint destruction and infiltration of inflammatory cells in the synovium of mice fed TG plants as compared to joints from mice in the control groups fed
WT plants (Figure 5a). The overall tissue destruction, as assessed by synovitis index and bone erosion index, was significantly lower in tolerogen fed mice compared to controls (Figure 5b). Hence, these histological data confirmed our clinical observations and demonstrated that feeding of TG plants expressing the CTA1 (R7K)-COL-DD tolerogen had a strong protective effect on CIA disease progression.

\section{Discussion}

The present proof-of-principle study provides evidence that edible plants can be used to successfully treat autoimmune diseases and more specifically autoimmune arthritis. We report here that mice fed with TG $A$. thaliana expressing the CTA1(R7K)-COL-DD tolerogen prevented $\mathrm{CIA}$ in $10-20 \%$ of the mice and that those afflicted had significantly milder symptoms. Overall tissue destruction, as shown by inflammatory scores of the synovia as well as bone erosion index, was significantly lower in the treated mice. The results from our study shed light on the potential to treat patients directly by feeding edible plants as a salad or, perhaps, allowing the plants to be reformulated into tablets for oral treatment of disease. Nevertheless, we achieved this by combining immunomodulation with effective targeting of disease-associated peptides in a plant expression vector that provided shielding of the tolerogen from degradation. In this way, the combination represents a means to overcome the requirement for large amounts of peptides, which has hampered success and lowered enthusiasm for edible plants as treatment for 
(a)
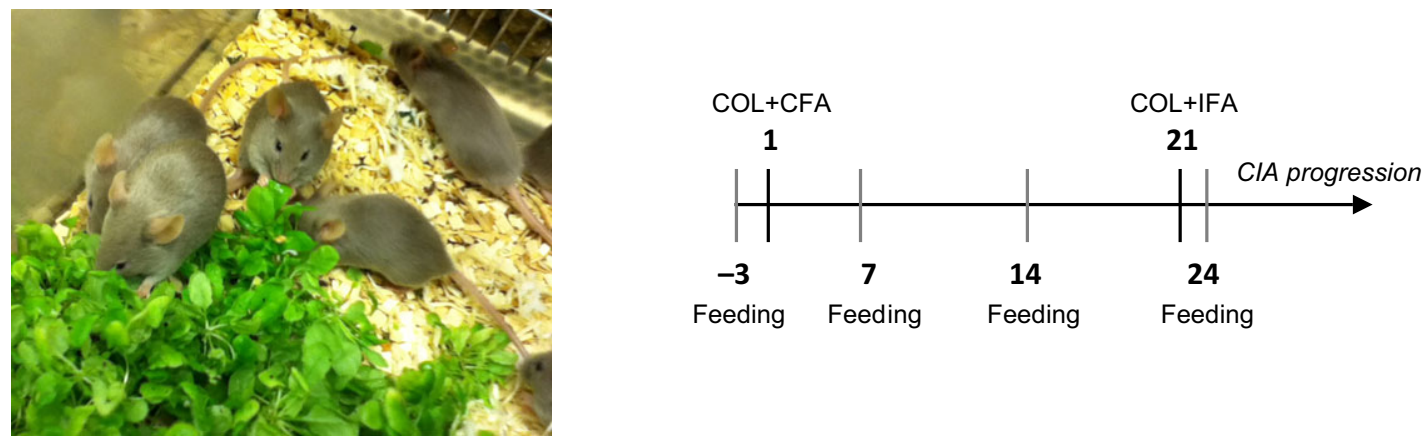

(b)

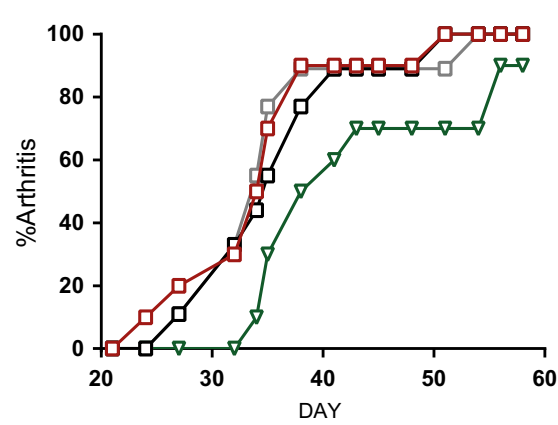

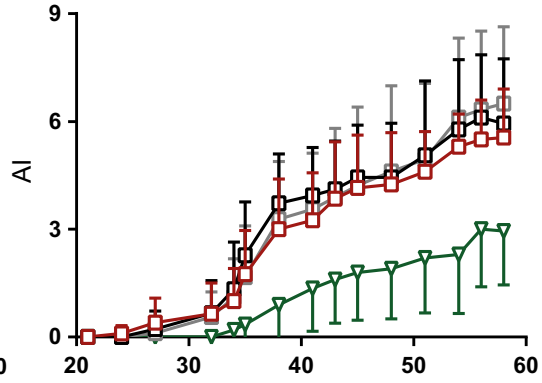

(c)

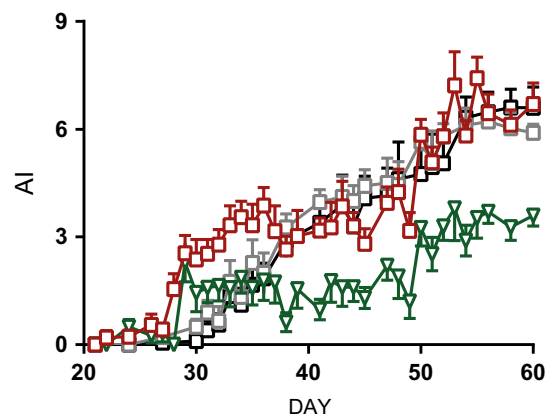

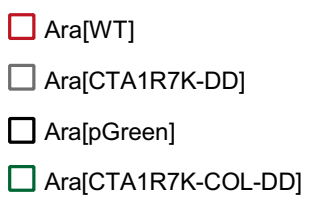

AUC

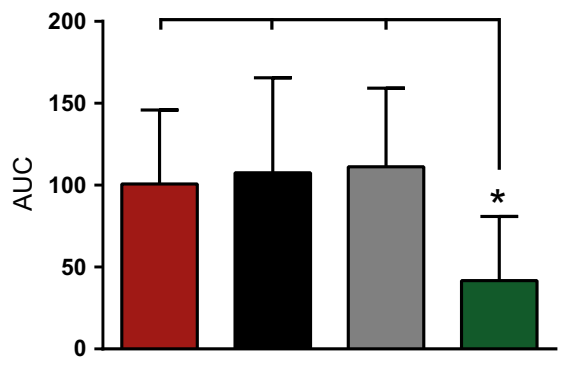

Arthritis index

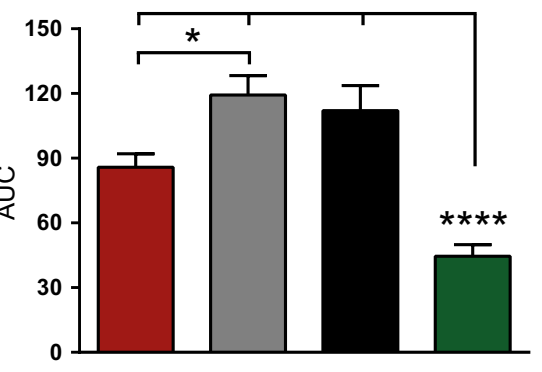

Figure 2 Feeding transgenic Arabidopsis thaliana plants to mice protects against CIA disease. DBA/1 mice were divided into groups of ten and fed approximately $70 \mathrm{~g}$ of WT or TG A. thaliana according to the experiment protocol (a). After the booster dose of CII in IFA on day 21, the mice were under observation for onset of CIA and scored regularly to determine the progression of disease. AUC valued were calculated for statistical analyses (b-c). Data are shown from one representative experiment and shown as mean $\pm \mathrm{Cl}(\mathrm{b})$ or from five pooled experiments $(n=20-50)$ and shown as mean \pm SEM (c). Statistical significance was determined by a one-way ANOVA, where * $P<0.05$ and $* * * * P<0.0001$

autoimmune diseases in the past (Garcia et al., 1999; Khare et al., 1995; Park et al., 2009; al-Sabbagh et al., 1994; Thurau et al., 1997; Zhu et al., 2007).

In this study, we estimated that as little as $40 \mu \mathrm{g}$ of the COLpeptide in total was sufficient to achieve significant clinical effects in the CIA mouse model. This was calculated on the basis of $2.5 \%$ of TSP of CTA1(R7K)-COL-DD fusion protein in TG A. thaliana plants. Compared to previous studies, this dose is low, especially considering that other studies have used 2 weeks of daily feeding of tolerogenic plants, amounting to, at least, a total of $350 \mu \mathrm{g}$ of peptide (Hashizume et al., 2008; lizuka et al., 2014). However, it should be noted that because mice were fed ad libitum, some animals may have ingested a higher dose and some a lower dose, which could have impacted on the outcome of our study. This is a frequently encountered problem with edible vaccines and has been discussed previously (Lal et al., 2007). As a generalization, though, we can claim that the individual mice ingested a dose that, at the group level, was sufficient to give a statistically significant reduction in disease score (IL-6) or tissue destruction (MMP-3). Individual variability is always a problem in any study like this, but given that we could undertake a controlled study with feeding exact doses of the plants, we may, in fact, have achieved an even better result. In our previous intranasal treatment protocols, a dose of $5 \mu \mathrm{g}$ fusion protein corresponding to $0.2 \mu \mathrm{g}$ of COL-peptide gave significant protective effects against CIA disease [6]. Although complete disease prevention was observed in more animals $(60 \%$ reduction compared to 10-20\%) following intranasal treatment, the effect on arthritic index and tissue destruction after feeding the tolerogen was roughly a $50 \%$ reduction and quite comparable to that seen following intranasal administration (Hasselberg et al., 2009). This means that the fusion protein was well protected in the plant and effectively taken up by the GALT (Rigano et al., 2003). 

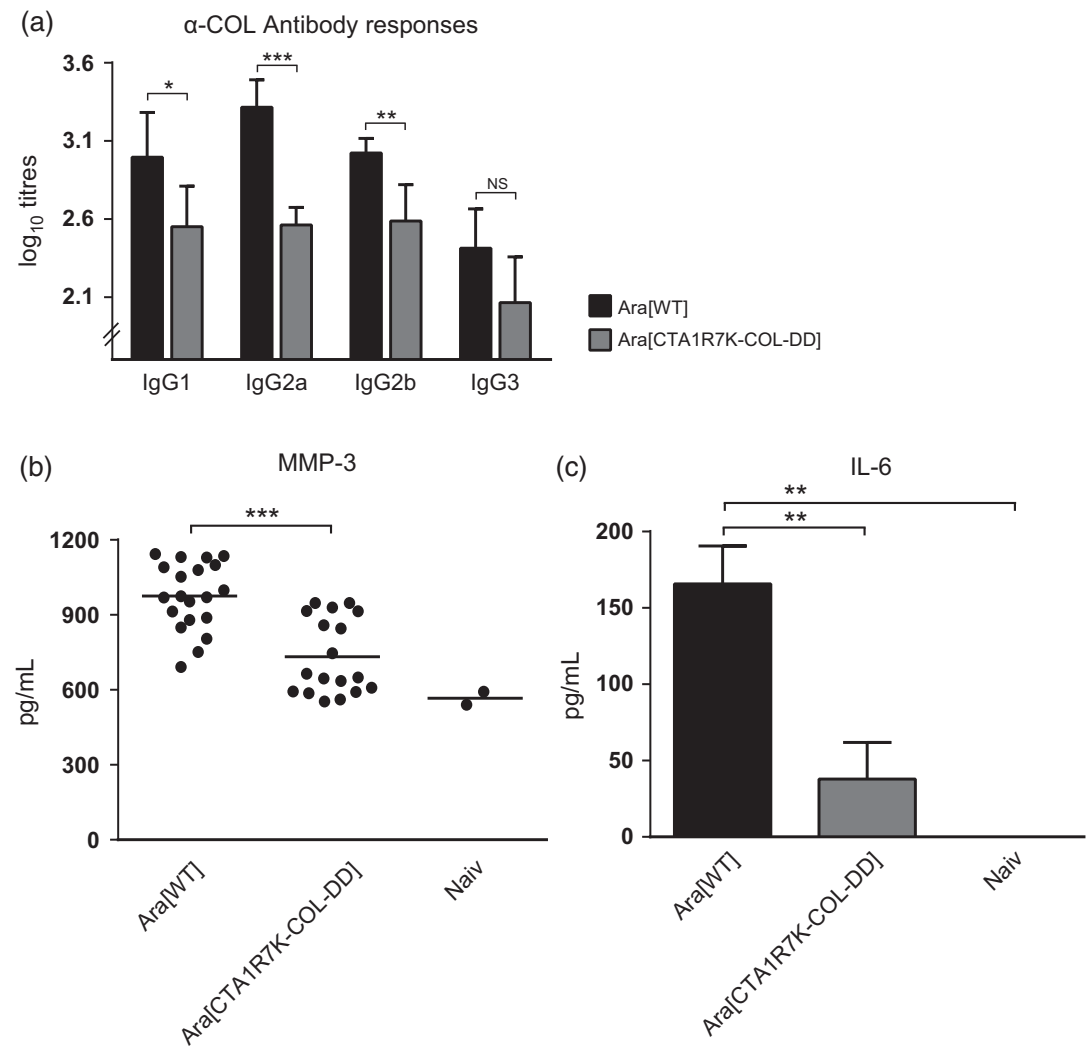

\begin{abstract}
Figure 3 Systemic responses to $\mathrm{CIA}$ are reduced in plant fed mice. Serum was collected from all remaining mice at the end of the experiment. CIIspecific antibody levels of the different lgG subclasses were determined by ELISA, and values are shown as mean $\log _{10}$-titres $\pm S D$ from one representative experiment $(n=4-5)$ (a). Levels of MMP-3 (b) and IL-6 (c) in serum are given as individual values and the mean \pm SEM from pooled experiments $(b-c)$. Statistical significance was calculated using an unpaired Student's t-test (a) or a one-way ANOVA $(b-c)$, where * $P<0.05$ and $* * P<0.01$ and $* * * P<0.001$.
\end{abstract}

A predominant fraction of the fusion protein was found in the soluble fraction of the plant, but the insoluble fraction also hosted some fusion protein which could be trapped in inclusion bodies (IBs). This may, in fact, have contributed to shielding and effective uptake of our tolerogen in the intestine. (Howe et al., 2014; O'Hagan, 1996). Thus, although IBs traditionally represent a challenge in other biotechnological applications, in this case, it might be beneficial instead (Villaverde et al., 2012). Our study underscores the efficiency by which oral treatment with tolerogen-expressing edible plants can convey protection against autoimmune diseases. Furthermore, this study shows that DD-targeting and CTA1(R7K)-immunomodulation are important features of the tolerogen, also when given orally. Of note, in previous studies, we have shown that an equimolar dose of peptide alone given intranasally has no toleranceinducing effect (Hasselberg et al., 2010). Moreover, Hashizume et al. expressed the CII250-270 peptide in glutelin as 4 tandem repeats in transgenic rice seeds and after feeding $25 \mu \mathrm{g}$ of peptide daily for 2 weeks to mice they reported a suppression of collagen-specific lgG2a antibody levels, although these were not significant (Hashizume et al., 2008). This suggests that the peptide was taken up, but under suboptimal conditions and, thus, could not interfere effectively with ongoing immune responses. As our protein expression (2.5\% of TSP) was comparable to that reported in many other studies, we believe the DD-targeting and CTA1(R7K) immunomodulation strongly contribute to explaining the difference between previous, less promising, attempts to tolerize against autoimmune diseases using edible plants and the success of this study (Avesani et al., 2010). The use of CTB as a delivery vehicle for protein or peptide for oral tolerization is well documented, for example, when expressed in potatoes or lettuce (Arakawa et al., 1998; Ruhlman et al., 2007). In support of the notion that adjuvants greatly augment the tolerizing effect of edible plants, Ruhlmann et al. showed in the type 1 diabetes NOD mouse model that feeding CTB-proinsulin could lead to significant immunosuppression and less pancreatic insulitis, although this effect may be due to Th2-skewing rather than the induction of Tregs (Ruhlman et al., 2007).

Our clinical observations of milder arthritis following TG plant feeding were also validated by decreased levels of disease biomarkers in serum, indicative of a reduced severity of $\mathrm{CIA}$. These biomarkers involved MMP-3 and IL-6. Whereas IL-6 is a general marker for inflammation and a reduction of IL-6 also reduces the differentiation of Th17 effector cells, MMP-3 levels in serum have been found to closely relate to RA disease intensity and joint destruction (Kolls and Linden, 2004; Seeuws et al., 2010). We monitored MMP-3 levels in TG plant fed mice, and the reduction was found to be almost to the level observed in healthy naive mice. The low level of expression of the MMP-3 biomarker also correlated with significantly reduced synovitis and bone erosion scores, in agreement with our clinical scoring. We also observed a decreased serum level of specific total lgG antibodies. An extended analysis demonstrated that both IgG1- and IgG2aspecific antibody responses were reduced, suggesting the suppression of effector functions in both the Th1 and Th2 CD4 T-cell subsets. Moreover, we observed increased IL-10 production concomitant with decreased Th1 (IFNy), Th2 (IL-13) and Th17 (IL-17 \& GM-CSF) cytokine responses in supernatants from splenocytes exposed to recall antigen in vitro. This finding agrees well with our previous observation of induction of IL-10-producing systemic Tregs after intranasal treatment with the tolerogen (Hasselberg et al., 2010). Thus, we conclude that also after oral administration of plant-expressed tolerogen, we achieved induction of IL-10 producing Tregs. In our previous study, these were identified as $\operatorname{Tr} 1$ cells because they failed to express Foxp3, but 
Figure 4 Suppressed CD4 T-cell responses to CIA in mice fed transgenic plants. At the end of the experiment, lymphocytes from spleen or popliteal lymphnodes were isolated from mice that had not yet been euthanized and restimulated with recall $\mathrm{COL}_{259-274}$ peptide in vitro. Proliferation was assessed after 72 hours by thymidine incorporation (a), and supernatants were collected and analysed for cytokine content (b). Furthermore, FoxP3+ CD4 T cells in peripheral blood were quantified by FACS (c). Proliferation data (a) are summarized from one representative experiment of one (popLN) or four (SP) and shown as mean \pm SEM. Relative cytokine production in TG plant fed mice vs WT plant fed mice (b) is pooled data from two to four experiments and shown as mean \pm SEM. Representative FoxP3+ CD4 FACS plots (c) are shown and summarized as mean \pm SD from one of two independent experiments with similar findings. Statistical significance was measured by Student's t-test (a-b) or a one-way ANOVA (c), where * $P<0.05$ and $* * P<0.01$ and $* * * P<0.001$.
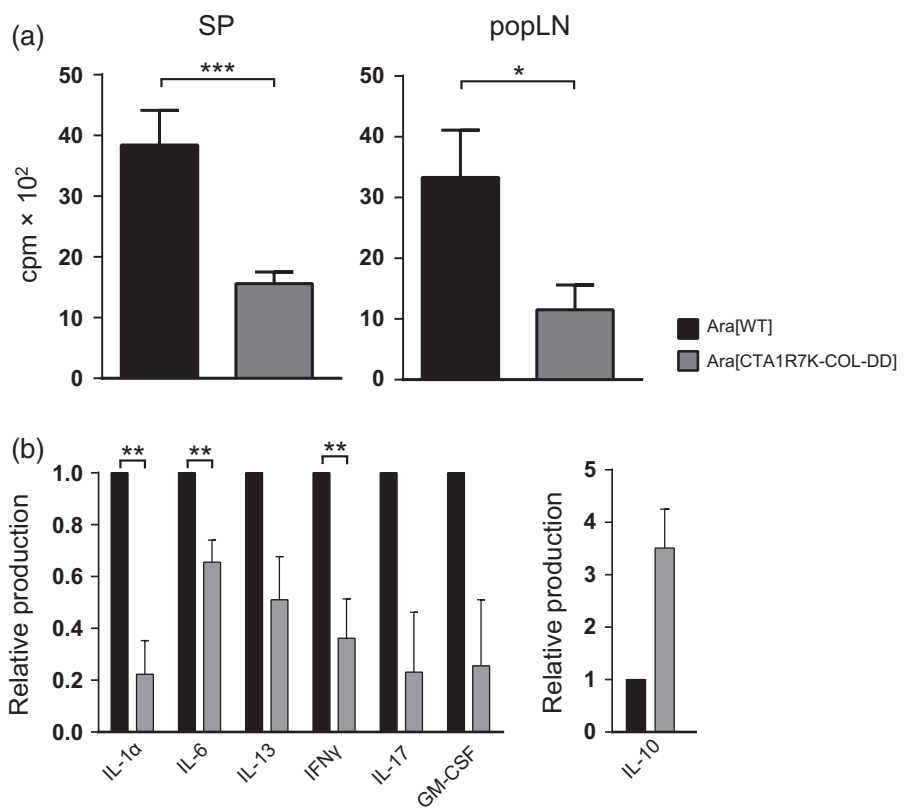

(c)
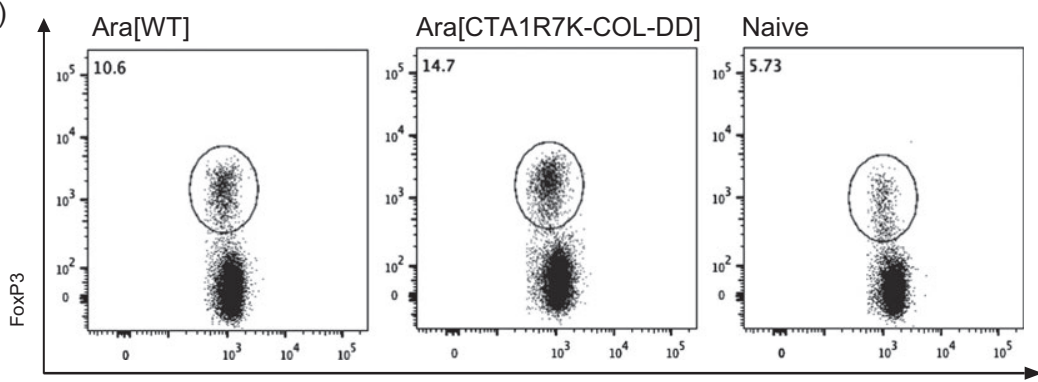

$\mathrm{CD}$
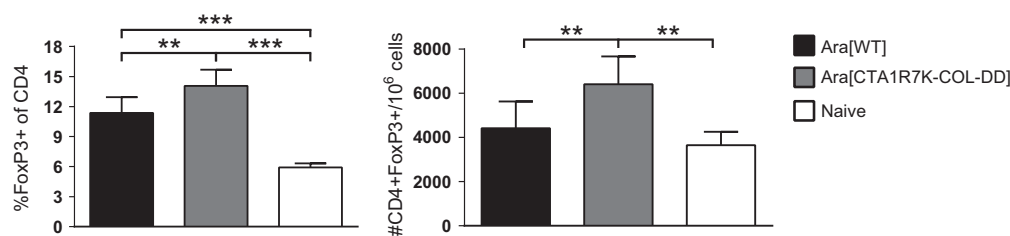

several new phenotypic markers, such as CD49b and lymphocyte activation gene 3 (LAG-3) could have been used to better identify Tr1 cells (Gagliani et al., 2013). Nevertheless, in the present study, we also observed an increase in circulating Foxp3 $3^{+}$CD4 T cells after feeding tolerogenic plants. However, we have not yet investigated whether they were COL-specific, inducible or natural Tregs, and whether they were able to exert suppression. Interestingly, in a recent study, feeding of plants expressing haemophilia B factor IX and a Foxp3 ${ }^{-}$Treg CD4 T-cell population expressing latency associated protein $3\left(\mathrm{LAP}^{+}\right)$were shown to exert oral tolerance (Sherman et al., 2014; Wang et al., 2015). Hence, the Treg subsets involved in oral tolerance after feeding recombinant edible plants may be complex and involve not only classical Foxp3 ${ }^{+}$CD4 T cells, but also Foxp3 ${ }^{-}$Tr1 cells and LAP ${ }^{+}$ Tregs that produce not only IL-10, but also TGF $\beta$.

In conclusion, we have greatly expanded on the clinical potential of our patented CTA1(R7K)-COL-DD fusion protein, which expressed in edible plants and fed to mice protected against CIA and ameliorated symptoms and tissue destruction. To our knowledge, this is the first study to clearly demonstrate how edible plants can successfully be used to treat autoimmune arthritis. The study also serves as an example of how to combine fusion protein targeting and immunomodulation to achieve robust tolerance even with very low doses of the disease-relevant peptides. Our results convey optimism that edible plants, as for example, a salad, or a plant-derived tablet with the tolerogen, may be effective in treating RA and possibly also other autoimmune diseases. Needless to say, edible plants still represent a very cost-effective and safe treatment strategy for delivery of tolerogens for treatment of autoimmune diseases. Finally, it should be mentioned that although clinical use of this strategy is attractive several limitations need to be addressed. Patients will most likely not receive treatment until they present with significant symptoms and the disease is well underway. The therapeutic impact of edible plants expressing CTA1(R7K)-COL-DD in RA patients, therefore, awaits to be assessed. Moreover, as only one Cll dominant peptide was used (aa 259-274), it is unknown whether a therapy based on this will be effective, given that epitope spreading is a well-known phenomenon in autoimmune diseases. However, it is well established that human HLA-DRB $1 * 04$ alleles can present this epitope in its glycosylated as well as nonglycosy- 
(a)
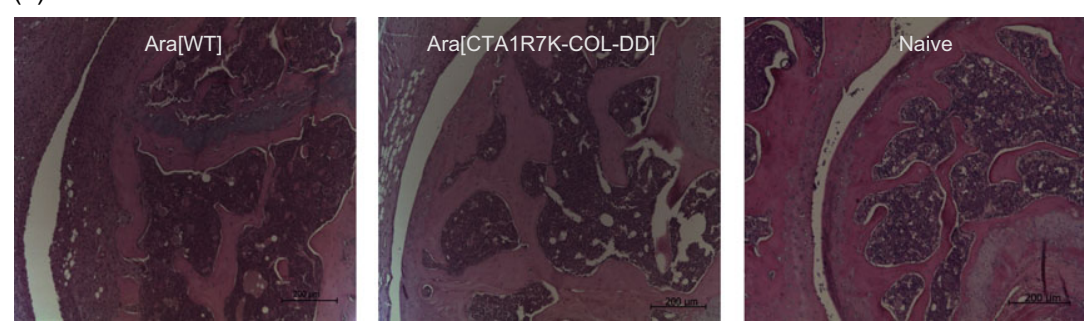

(b) Inflammation
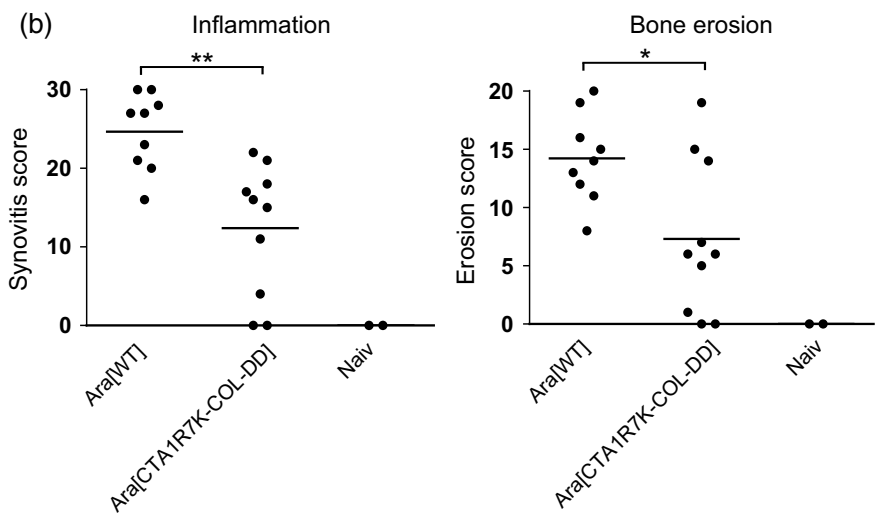

Figure 5 Feeding tolerance-inducing plants protects against CIA disease and tissue destruction. When mice were euthanized, before or at the termination of the experiment, joints were collected and stained for haematoxylin and eosin (a). Lymphocyte infiltrates and cartilage destruction were estimated to determine the severity of synovitis, inflammation and bone erosion (b). Data from one of two independent experiments is shown as a scatter dot plot, and statistical significance was determined by a oneway ANOVA, where $* P<0.05$ and $* * P<0.01$. lated forms and that recognition was seen in 7 of 10 patients and that reactivity in the same individual over several years appeared to persist (Snir et al., 2012). These are all favourable elements that speak to the advantage of the CTA1R7K-COL-DD tolerogen in edible plants for treatment of RA patients.

\section{Experimental procedures}

\section{Production of transgenic Arabidopsis thaliana}

Transgenic plants were produced by a simplified Agrobacteriummediated floral dip method as described by Clough and Bent (Clough and Bent, 1995). The transgene CTA1(R7K)-COL-DD was assembled in the pGreen vector (http://www.pgreen.ac.uk) (Hellens et al., 2000) kindly provided by Dr. P. Mullineaux, John Innes Centre and the Biotechnology and Biological Sciences Research Council (Norwich Research Part, UK). The expression cassette contained a constitutive plant promotor CaMV 35 S and a CaMV polyA termination sequence, separated by a multicloning site. The vector was linearized at the multicloning site by Smal restriction enzyme and used for cloning of the transgene (Figure 1a). The pGreen/CTA1(R7K)-COL-DD vector was used to transform Agrobacterium tumefaciens strain EHA105 by electroporation. Positive clones were selected on Luria Broth (LB) medium supplemented with kanamycin $(50 \mu \mathrm{g} / \mathrm{mL})$ and tetracyclin $(5 \mu \mathrm{g} /$ $\mathrm{mL}$ ) and verified by PCR and sequencing (ABI PRISM 310 Genetic Analyser; Applied Biosystems, Waltham, MA). Four-week-old A. thaliana plants (ecotype Col-0; The European Arabidopsis Stock Centre, Loughborough, UK) were used for transformation of the nuclear genome with the Agrobacterium clone containing the transgene construct (Clough and Bent, 1995). Seeds were harvested from the plants that had undergone floral dip and spread onto agar plates with Murashige and Skoog medium supplemented with $10 \mu \mathrm{g} / \mathrm{mL}$ herbicide BASTA (Riedel-de Haën, Seelze, Germany) and $400 \mu \mathrm{g} / \mathrm{mL}$ cephotaxime (Sigma-Aldrich, Stockholm, Sweden). Resistant seedlings were transferred to potting mix (soil: perlite: vermiculite, 1:1:1) for analysis, self- pollination and seed production. The seeds obtained from individual plants producing 100\% BASTA-resistant progeny were used for feeding experiments after additional confirmation by $P C R$, sequencing and Western blot analysis.

\section{Expression analysis of the transgenic plants}

Cytoplasmic expression by the TG plants was analysed using immunoblotting. Arabidopsis tissues were ground in an extraction buffer containing $50 \mathrm{~mm}$ Tris ( $\mathrm{pH}$ 7.5) with or without $8 \mathrm{M}$ urea containing $1 \mu \mathrm{M}$ protease inhibitor PMSF (Sigma, Stockholm, Sweden). After centrifugation, the samples were separated by SDS-PAGE and blotted onto nitrocellulose membrane Hybond-C (GE Healthcare, Uppsala, Sweden). The membranes were blocked with $1 \%$ BSA in TBS-T $(0.02 \mathrm{M}$ Tris- $\mathrm{HCl}, 0.15 \mathrm{M} \mathrm{NaCl}, 0.1 \%$ Tween 20, pH 7.5) and hybridized with $\alpha$-CTA1-DD (Agrisera, Vännäs, Sweden) antibodies. The protein: primary antibody complexes were then detected by alkaline phosphatase (AP)conjugated anti-chicken or anti-rabbit antibodies (Promega, Madison, WI) and visualized with nitroblue tetrazolium chloride (NBT) and 5-bromo-4-chloro-3-indolyl phosphate (BCIP; Promega). Protein extract from nontransformed wild-type (WT) Arabidopsis was included in all runs as a negative control and recombinant CTA1-DD protein (Agren et al., 1997) was used as a positive control for semiquantitative analysis of the CTA1(R7K)COL-DD expression in plants. Total soluble protein (TSP) in the TG plants was extracted using $50 \mathrm{~mm}$ Tris (pH 7.5) and quantified with the Bradford method/Bio-Rad protein assay (Bio-Rad Laboratories, Solna, Sweden) using bovine serum albumin (BSA) for construction of the standard curve.

\section{Plant tissue subfractionation}

Arabidopsis leaves were homogenized in ice-cold MES buffer $(\mathrm{pH}$ 6.5) using a prechilled blender, and the homogenate was filtered using two layers of Miracloth (Calbiochem, San Diego, CA). The released chloroplasts were pelleted at $3000 \mathrm{~g}$, washed in $50 \mathrm{~mm}$ HEPES buffer ( $\mathrm{pH} 8$ ) and pelleted again at $5000 \mathrm{~g}$ three times. All 
steps were carried out at $4{ }^{\circ} \mathrm{C}$. The debris retained in the Miracloth was returned to the blender and the homogenization and filtering steps were repeated twice. The remaining material in the Miracloth was an insoluble fraction composed of cellular debris and inclusion bodies. This fraction was further extracted with $50 \mathrm{~mm}$ TRIS buffer ( $\mathrm{pH}$ 7.5) containing $8 \mathrm{~m}$ urea for analysis. After 3 washing steps and pelleting of the chloroplast fraction, the supernatants containing cytoplasmic soluble proteins were collected and concentrated using Amicon Ultra-10 filter device with a cut-off of $10 \mathrm{kDa}$ (Merck Millipore, Billerica, MA). All three fractions (chloroplasts, cytoplasmic soluble proteins, and insoluble fraction) were analysed for the presence of the recombinant protein by Western blotting

\section{Mice, feeding and induction of $\mathrm{CIA}$}

Male DBA/1 $\left(\mathrm{H}-2^{9}\right)$ mice, ages 8-12 weeks (Harlan, The Netherlands), were kept under specific pathogen-free conditions at the department of experimental medicine (University of Gothenburg). Mice were fed transgenic or wild-type $A$. thaliana plants ad libitum once every week for 4 weeks, corresponding to $70 \mathrm{~g}$ of material, and conventional food was removed $(<12$ h) on these occasions (Figure 2a). CIA was induced by a $100 \mu \mathrm{L}$ intradermal injection with $100 \mu \mathrm{g}$ of collagen type II (CII) from bovine tracheal cartilage (MD Biosciences, St Paul, MN) emulsified in an equal volume of complete Freunds adjuvant (CFA; 4 mg/mL Mycobacterium tuberculosis; MD Biosciences). On day 21, an intradermal booster injection with $100 \mu \mathrm{L}$ of $100 \mu \mathrm{g} \mathrm{Cll}$ emulsified in Freunds incomplete adjuvant (IFA; Sigma) was given, and disease progression was monitored for 40-60 days. CIA severity was determined as previously described (Brand et al., 2007; Tarkowski et al., 1999). Briefly, limbs were scored 0-3, where 0: no inflammation, 0.5 : toe or finger swelling, 1 : mild swelling or redness, 2: swelling and redness, 3: marked swelling, redness and/or ankylosis. Mice with a score $>6$ during examination were euthanized. Studies were approved by the Ethics Committee for Animal Experimentation, University of Gothenburg.

\section{Antibody determinations}

96-well, transparent, flat-bottomed plates (Nunc, Roskilde, Denmark) were coated with $10 \mu \mathrm{g} / \mathrm{mL}$ rat CII (Sigma-Aldrich) and blocked with $1 \%$ BSA/PBS. Serial threefold dilutions of individual serum samples were incubated overnight at $4{ }^{\circ} \mathrm{C}$. After washing with $0.1 \%$ BSA/PBS three times, either alkaline phosphataseconjugated goat antimouse IgG1-, IgG2a-, IgG2b- or IgG3 antibodies (Southern Biotechnology, Birmingham, AL) were added. The 4-nitrophenyl phosphate disodium salt hexahydrate (NP; $1 \mathrm{mg} / \mathrm{mL}$; Sigma) enzymatic reaction was analysed using a Tecan Sunrise ELISA Reader (Nordic Biolabs, Stockholm, Sweden). Anticollagen serum $\log _{10}$-titres were defined as the reciprocal serum dilution, giving an absorbance of $0.4 \mathrm{~nm}$ above background levels.

\section{Histological assessments}

Paws were treated with paraformaldehyde (Histolab, Gothenburg, Sweden) and embedded in paraffin, as described previously (Tarkowski et al., 1999). Tissue sections were stained with haematoxylin and eosin (Histolab) and an experienced pathologist performed a blinded examination of the tissues for the presence of synovial hypertrophy, pannus formation and bone erosion. Elbows, wrists, carpal joints, fingers, knees, ankles, tarsal joints and toes were scored for inflammation and erosion, where $1=$ mild, 2 = moderate and $3=$ severe synovitis. MMP3 levels were assessed in serum by a quantitative ELISA kit (R\&D Systems, Minneapolis, MN) according to manufacturer's instructions.

\section{In vitro culturing and cytokine assays}

Single-cell suspensions from spleen or popliteal lymph nodes $\left(1 \times 10^{6} / \mathrm{mL}\right)$ were cultured in 96-well, round-bottomed plates (Nunc) using Iscove's medium (Biochrom, Berlin, Germany) supplemented with $10 \%$ heat-inactivated foetal calf serum (FCS; Biochrom), $50 \mu \mathrm{m}$ 2-ME (Sigma-Aldrich), 1 mм 1-glutamine (Biochrom) and $50 \mu \mathrm{g} / \mathrm{mL}$ gentamicin (Sigma-Aldrich) for $72 \mathrm{~h}$ at $37{ }^{\circ} \mathrm{C}$ in $5 \% \mathrm{CO}_{2}$. Cells were incubated with or without $5 \mu \mathrm{m}$ of the CIl peptide COL (aa 259-274: GIAGFKGEQGPKGEPG; Agrisera) in triplicates, and after $72-\mathrm{h}, \mathrm{T}$-cell cytokine production in supernatants or proliferation, the addition of $1 \mathrm{mCi}[3 \mathrm{H}]-$ thymidine (PerkinElmer, Boston, MA) for $6 \mathrm{~h}$, was assessed. A beta-scintillation counter (Beckman Coulter, Turku, Finland) was used to measure [3H]-thymidine incorporation. Cytokines in supernatants or serum were determined using a mouse Th1/ Th2/Th17 cytometric bead array kit (eBioscience, Santa Clara, CA) according to the manufacturer's instructions.

\section{FACS analysis}

Peripheral blood was collected in di-potassium-EDTA-coated Microvette tubes (Sarstedt, Nümbrecht, Germany), and erythrocytes were lysed using BD Pharm Lyse ${ }^{\mathrm{TM}}$ lysing buffer (BD Biosciences, Stockholm, Sweden) and washed three times. Cells were resuspended in $0.1 \%$ BSA/PBS and incubated with the FCR blocking $A b(24 G 2)$ for 5 min before adding AlexaFluor 700conjugated $\alpha$-CD4 (BD Biosciences). Cells were then fixated and permeabilized using the FoxP3 staining buffer kit (eBioscience) and finally incubated with PE-conjugated $\alpha$-FoxP3 (eBioscience) before analysis.

\section{Statistical analysis}

As specified in the figure legends, an unpaired Student's t-test or a one-way ANOVA was used to calculate statistical significance. The analyses were performed using the Prism software (GraphPad, La Jolla, CA), and $P$-values $<0.05$ were considered statistically significant.

\section{Acknowledgements}

This study was supported by grants from the Swedish Cancerfoundation, Vetenskapsrådet Medicin, Strategiska Stiftelserna, Knut \& Alice Wallenbergs Stiftelse, AFA-försäkringar, EU projects in FP7 UniVacFlu and UNISEC.

\section{References}

Agren, L.C., Ekman, L., Lowenadler, B. and Lycke, N.Y. (1997) Genetically engineered nontoxic vaccine adjuvant that combines B cell targeting with immunomodulation by cholera toxin A1 subunit. J. Immunol. 158, 3936-3946. Arakawa, T., Yu, J., Chong, D.K., Hough, J., Engen, P.C. and Langridge, W.H. (1998) A plant-based cholera toxin B subunit-insulin fusion protein protects against the development of autoimmune diabetes. Nat. Biotechnol. 16, 934938.

Avesani, L., Bortesi, L., Santi, L., Falorni, A. and Pezzotti, M. (2010) Plant-made pharmaceuticals for the prevention and treatment of autoimmune diseases: where are we? Expert Rev. Vaccines 9, 957-969.

Barnett, M.L., Combitchi, D. and Trentham, D.E. (1996) A pilot trial of oral type II collagen in the treatment of juvenile rheumatoid arthritis. Arthritis Rheum. 39, 623-628 
Barnett, M.L., Kremer, J.M., St Clair, E.W., Clegg, D.O., Furst, D., Weisman, M., Fletcher, M.J., Chasan-Taber, S., Finger, E., Morales, A., Le, C.H. and Trentham, D.E. (1998) Treatment of rheumatoid arthritis with oral type I collagen. Results of a multicenter, double-blind, placebo-controlled trial. Arthritis Rheum. 41, 290-297.

Beyaert, R., Beaugerie, L., Van Assche, G., Brochez, L., Renauld, J.C., Viguier, M., Cocquyt, V., Jerusalem, G., Machiels, J.P., Prenen, H., Masson, P., Louis, E. and De Keyser, F. (2013) Cancer risk in immune-mediated inflammatory diseases (IMID). Mol. Cancer. 12, 98.

Bongartz, T., Sutton, A.J., Sweeting, M.J., Buchan, I., Matteson, E.L. and Montori, V. (2006) Anti-TNF antibody therapy in rheumatoid arthritis and the risk of serious infections and malignancies: systematic review and metaanalysis of rare harmful effects in randomized controlled trials. JAMA, 295 2275-2285

Brand, D.D., Kang, A.H. and Rosloniec, E.F. (2003) Immunopathogenesis of collagen arthritis. Springer Semin. Immunopathol. 25, 3-18.

Brand, D.D., Latham, K.A. and Rosloniec, E.F. (2007) Collagen-induced arthritis. Nat. Protoc. 2, 1269-1275.

Brooks, P.M. (2006) The burden of musculoskeletal disease-a global perspective. Clin. Rheumatol. 25, 778-781.

Cazzola, M., Antivalle, M., Sarzi-Puttini, P., Dell'Acqua, D., Panni, B. and Caruso, I. (2000) Oral type II collagen in the treatment of rheumatoid arthritis. A six-month double blind placebo-controlled study. Clin. Exp. Rheumatol. 18, 571-577.

Choy, E.H., Scott, D.L., Kingsley, G.H., Thomas, S., Murphy, A.G., Staines, N. and Panayi, G.S. (2001) Control of rheumatoid arthritis by oral tolerance. Arthritis Rheum. 44, 1993-1997.

Clough, S. and Bent, A. (1995) Floral dip: a simplified method for Agrobacterium-mediated transformation of Arabidopsis thaliana. Plant J. 16, 735-743

Faria, A.M. and Weiner, H.L. (2006) Oral tolerance: therapeutic implications for autoimmune diseases. Clin. Dev. Immunol. 13, 143-157.

Firestein, G.S. (2003) Evolving concepts of rheumatoid arthritis. Nature, 423, 356-361.

Gagliani, N., Magnani, C.F., Huber, S., Gianolini, M.E., Pala, M., Licona-Limon, P., Guo, B., Herbert, D.R., Bulfone, A., Trentini, F., Di Serio, C., Bacchetta, R., Andreani, M., Brockmann, L., Gregori, S., Flavell, R.A. and Roncarolo, M.G. (2013) Coexpression of CD49b and LAG-3 identifies human and mouse T regulatory type 1 cells. Nat. Med. 19, 739-746.

Garcia, G., Komagata, Y., Slavin, A.J., Maron, R. and Weiner, H.L. (1999) Suppression of collagen-induced arthritis by oral or nasal administration of type II collagen. J. Autoimmun. 13, 315-324

Hashizume, F., Hino, S., Kakehashi, M., Okajima, T., Nadano, D., Aoki, N. and Matsuda, T. (2008) Development and evaluation of transgenic rice seeds accumulating a type II-collagen tolerogenic peptide. Transgenic Res. 17 1117-1129.

Hasselberg, A., Schon, K., Tarkowski, A. and Lycke, N. (2009) Role of CTA1R7K-COL-DD as a novel therapeutic mucosal tolerance-inducing vector for treatment of collagen-induced arthritis. Arthritis Rheum. 60, 1672-1682

Hasselberg, A., Ekman, L., Yrlid, L.F., Schon, K. and Lycke, N.Y. (2010) ADPribosylation controls the outcome of tolerance or enhanced priming following mucosal immunization. J. Immunol. 184, 2776-2784.

Hellens, R.P., Edwards, E.A., Leyland, N.R., Bean, S. and Mullineaux, P.M. (2000) pGreen: a versatile and flexible binary Ti vector for Agrobacteriummediated plant transformation. Plant Mol. Biol. 42, 819-832.

Howe, S.E., Lickteig, D.J., Plunkett, K.N., Ryerse, J.S. and Konjufca, V. (2014) The uptake of soluble and particulate antigens by epithelial cells in the mouse small intestine. PLOS ONE 9, e86656.

Ichim, T.E., Zheng, X., Suzuki, M., Kubo, N., Zhang, X., Min, L.R., Beduhn, M.E., Riordan, N.H., Inman, R.D. and Min, W.P. (2008) Antigen-specific therapy of rheumatoid arthritis. Expert Opin. Biol. Ther. 8, 191-199.

lizuka, M., Wakasa, Y., Tsuboi, H., Asashima, H., Hirota, T., Kondo, Y., Matsumoto, I., Takaiwa, F. and Sumida, T. (2014) Suppression of collageninduced arthritis by oral administration of transgenic rice seeds expressing altered peptide ligands of type II collagen. Plant Biotechnol. J. 12, 1143-1152. Jabbal-Gill, I. (2010) Nasal vaccine innovation. J. Drug Target. 18, 771-786.
Khare, S.D., Krco, C.J., Griffiths, M.M., Luthra, H.S. and David, C.S. (1995) Oral administration of an immunodominant human collagen peptide modulates collagen-induced arthritis. J. Immunol. 155, 3653-3659.

Kolls, J.K. and Linden, A. (2004) Interleukin-17 family members and inflammation. Immunity 21, 467-476.

Lal, P., Ramachandran, V.G., Goyal, R. and Sharma, R. (2007) Edible vaccines: current status and future. Indian J. Med. Microbiol. 25, 93-102.

Limaye, A., Koya, V., Samsam, M. and Daniell, H. (2006) Receptor-mediated oral delivery of a bioencapsulated green fluorescent protein expressed in transgenic chloroplasts into the mouse circulatory system. FASEB J. 20, 959961.

Ma, S.W., Zhao, D.L., Yin, Z.Q., Mukherjee, R., Singh, B., Qin, H.Y., Stiller, C.R. and Jevnikar, A.M. (1997) Transgenic plants expressing autoantigens fed to mice to induce oral immune tolerance. Nat. Med. 3, 793-796.

McInnes, I.B. and Schett, G. (2007) Cytokines in the pathogenesis of rheumatoid arthritis. Nat. Rev. Immunol. 7, 429-442.

O'Hagan, D.T. (1996) The intestinal uptake of particles and the implications for drug and antigen delivery. J. Anat. 189(Pt 3), 477-482.

Okada, H., Kuhn, C., Feillet, H. and Bach, J.F. (2010) The 'hygiene hypothesis' for autoimmune and allergic diseases: an update. Clin. Exp. Immunol. 160, $1-9$.

Park, K.S., Park, M.J., Cho, M.L., Kwok, S.K., Ju, J.H., Ko, H.J., Park, S.H. and Kim, H.Y. (2009) Type II collagen oral tolerance; mechanism and role in collagen-induced arthritis and rheumatoid arthritis. Mod. Rheumatol. 19, 581-589.

Rigano, M.M., Sala, F., Arntzen, C.J. and Walmsley, A.M. (2003) Targeting of plant-derived vaccine antigens to immunoresponsive mucosal sites. Vaccine 21, 809-811.

Ruhlman, T., Ahangari, R., Devine, A., Samsam, M. and Daniell, H. (2007) Expression of cholera toxin B-proinsulin fusion protein in lettuce and tobacco chloroplasts-oral administration protects against development of insulitis in non-obese diabetic mice. Plant Biotechnol. J. 5, 495-510.

al-Sabbagh, A., Miller, A., Santos, L.M. and Weiner, H.L. (1994) Antigen-driven tissue-specific suppression following oral tolerance: orally administered myelin basic protein suppresses proteolipid protein-induced experimental autoimmune encephalomyelitis in the SJL mouse. Eur. J. Immunol. 24, 2104 2109.

Salliot, C., Dougados, M. and Gossec, L. (2009) Risk of serious infections during rituximab, abatacept and anakinra treatments for rheumatoid arthritis: meta-analyses of randomised placebo-controlled trials. Ann. Rheum. Dis. 68, 25-32.

Seeuws, S., Jacques, P., Van Praet, J., Drennan, M., Coudenys, J., Decruy, T., Deschepper, E., Lepescheux, L., Pujuguet, P., Oste, L., Vandeghinste, N., Brys, R., Verbruggen, G. and Elewaut, D. (2010) A multiparameter approach to monitor disease activity in collagen-induced arthritis. Arthritis. Res. Ther. 12 R160.

Sherman, A., Su, J., Lin, S., Wang, X., Herzog, R.W. and Daniell, H. (2014) Suppression of inhibitor formation against FVIII in a murine model of hemophilia $A$ by oral delivery of antigens bioencapsulated in plant cells. Blood, 124, 1659-1668.

Smolen, J.S. and Aletaha, D. (2015) Rheumatoid arthritis therapy reappraisal: strategies, opportunities and challenges. Nat. Rev. Rheumatol. 11, 276289.

Snir, O., Backlund, J., Bostrom, J., Andersson, I., Kihlberg, J., Buckner, J.H., Klareskog, L., Holmdahl, R. and Malmstrom, V. (2012) Multifunctional T cell reactivity with native and glycosylated type II collagen in rheumatoid arthritis. Arthritis Rheum. 64, 2482-2488.

Tarkowski, A., Sun, J.B., Holmdahl, R., Holmgren, J. and Czerkinsky, C. (1999) Treatment of experimental autoimmune arthritis by nasal administration of a type II collagen-cholera toxoid conjugate vaccine. Arthritis Rheum. 42, 16281634.

Thurau, S.R., Chan, C.C., Nussenblatt, R.B. and Caspi, R.R. (1997) Oral tolerance in a murine model of relapsing experimental autoimmune uveoretinitis (EAU): induction of protective tolerance in primed animals. Clin. Exp. Immunol. 109, 370-376.

Trentham, D.E., Townes, A.S. and Kang, A.H. (1977) Autoimmunity to type ॥ collagen an experimental model of arthritis. J. Exp. Med. 146, 857-868. 
Trentham, D.E., Dynesius-Trentham, R.A., Orav, E.J., Combitchi, D., Lorenzo, C., Sewell, K.L., Hafler, D.A. and Weiner, H.L. (1993) Effects of oral administration of type II collagen on rheumatoid arthritis. Science, 261, 1727-1730.

Villaverde, A., Garcia-Fruitos, E., Rinas, U., Seras-Franzoso, J., Kosoy, A., Corchero, J.L. and Vazquez, E. (2012) Packaging protein drugs as bacterial inclusion bodies for therapeutic applications. Microb. Cell Fact. 11, 76

Wang, X., Su, J., Sherman, A., Rogers, G.L., Liao, G., Hoffman, B.E., Leong, K.W., Terhorst, C., Daniell, H. and Herzog, R.W. (2015) Plant-based oral tolerance to hemophilia therapy employs a complex immune regulatory response including LAP+CD4+ T cells. Blood, 125, 2418-2427.

Weiner, H.L., da Cunha, A.P., Quintana, F. and Wu, H. (2011) Oral tolerance. Immunol. Rev. 241, 241-259.

Zhu, P., Li, X.Y., Wang, H.K., Jia, J.F., Zheng, Z.H., Ding, J. and Fan, C.M. (2007) Oral administration of type-II collagen peptide 250-270 suppresses specific cellular and humoral immune response in collagen-induced arthritis. Clin. Immunol. 122, 75-84. 